\title{
Intervention to improve the quality of antimicrobial prescribing for urinary tract infection: a cluster randomized trial
}

\author{
Akke Vellinga PhD, Sandra Galvin PhD, Sinead Duane PhD, Aoife Callan PhD, Kathleen Bennett PhD, \\ Martin Cormican MD, Christine Domegan PhD, Andrew W. Murphy MD
}

See also page 94 and www.cmaj.ca/lookup/doi/10.1503/cmaj.151103

Competing interests: Subsequent to this study, Aoife Callan became an employee of Novartis Ireland. No other competing interests were declared.

This article has been peer reviewed.

Accepted: Sept. 28, 2015 Online: Nov. 16, 2015

Correspondence to: Akke Vellinga, akke.vellinga@nuigalway.ie

CMAJ 2016. DOI:10.1503/ cmaj.150601

\begin{abstract}
Background: Overuse of antimicrobial therapy in the community adds to the global spread of antimicrobial resistance, which is jeopardizing the treatment of common infections.

Methods: We designed a cluster randomized complex intervention to improve antimicrobial prescribing for urinary tract infection in Irish general practice. During a 3-month baseline period, all practices received a workshop to promote consultation coding for urinary tract infections. Practices in intervention arms $A$ and $B$ received a second workshop with information on antimicrobial prescribing guidelines and a practice audit report (baseline data). Practices in intervention arm B received additional evidence on delayed prescribing of antimicrobials for suspected urinary tract infection. A reminder integrated into the patient management software suggested first-line treatment and, for practices in $\operatorname{arm} B$, delayed prescribing. Over the 6-month intervention, practices in arms A

and $B$ received monthly audit reports of antimicrobial prescribing.

Results: The proportion of antimicrobial prescribing according to guidelines for urinary tract infection increased in arms $A$ and $B$ relative to control (adjusted overall odds ratio [OR] 2.3, 95\% confidence interval [CI] 1.7 to 3.2; arm A adjusted OR 2.7, 95\% Cl 1.8 to 4.1; arm B adjusted OR 2.0, 95\% Cl 1.3 to 3.0). An unintended increase in antimicrobial prescribing was observed in the intervention arms relative to control (arm A adjusted OR 2.2, $95 \% \mathrm{Cl} 1.2$ to 4.0 ; arm B adjusted OR 1.4, $95 \% \mathrm{Cl} 0.9$ to 2.1 ). Improvements in guidelinebased prescribing were sustained at 5 months after the intervention.

Interpretation: A complex intervention, including audit reports and reminders, improved the quality of prescribing for urinary tract infection in Irish general practice. Trial registration: ClinicalTrials.gov, no. NCT01913860
\end{abstract}

$\mathrm{I}$ $\mathrm{n}$ its recent global report on antimicrobial resistance, the World Health Organization warned that widespread resistance is not just a future threat but a present-day reality. Many of the current treatment options for common infections are becoming less effective. ${ }^{1,2}$ The World Health Organization pointed out that tackling antimicrobial resistance requires a multisectoral approach involving patients, health care workers, policy-makers and industry. ${ }^{2}$

With the Supporting the Improvement and Management of Prescribing (SIMPle) study, we aimed to improve antimicrobial prescribing for urinary tract infection in primary care through a multifaceted complex intervention with interactive, multimedia and electronic components integrated into routine care. ${ }^{3}$ In this article, we report on the quality of prescribing measured against national guidelines for the prescribing of antimicrobials. $^{4}$

\section{Methods}

The study protocol was described previously. ${ }^{3}$ The SIMPle study was a 3-armed complex intervention, with practice-level randomization. Eligible practices were members of the Irish Primary Care Research Network that were using a particular type of patient management software. The Irish Primary Care Research Network is an established national research network of general practices. Of the 32 eligible practices invited by letter, 30 confirmed their participation in a follow-up phone call. Upon confirmation, each 
practice was sequentially assigned to 1 of the 3 study arms, according to a computer-generated randomization schedule.

\section{Intervention}

The intervention, based on prior formative research, ${ }^{3}$ aimed to improve the quality of antimicrobial prescribing for urinary tract infection in primary care through a multifaceted complex intervention with interactive, multimedia and electronic components (Table 1).

In phase 1 (baseline data collection), all general practitioners from each participating practice were invited to a coding workshop, during which the SIMPle study was explained, including the importance of consultation coding for the generation of audit reports. Phase 2 began with an interactive workshop, with differing content based on the intervention arm. Practices in intervention arm A received information on national guidelines for antimicrobial prescribing, and the first practice audit report (phase 1 data) was discussed. Practices in intervention arm $\mathrm{B}$ received the same information as those in arm A, along with additional evidence to support delaying antimicrobial prescriptions for suspected urinary tract infection. For practices in both intervention arms, whenever a consultation was coded as urinary tract infection, a reminder outlining the guidelines (including a link to the website www. antibioticprescribing.ie) appeared. For practices in $\operatorname{arm} \mathrm{B}$, the reminder also urged the physician to consider delayed prescribing. Practices in the intervention arms received a monthly audit of antimicrobial prescribing for urinary tract infection (by email). To standardize the intervention, control practices received a workshop focused on the coding routine. In phase 3 (intervention arms only), a multimedia application was introduced, which included a game for children and an infomercial for adults addressing antimicrobial awareness (Bug Run School Days), accessible to patients in the physicians' offices.
After the 6-month intervention period, control practices received all of the intervention materials, as well as their respective audit reports. Phase 4, the follow-up period, started at the end of the intervention and included a 5-month period of passive data collection to evaluate sustainability of any change in antimicrobial prescribing.

Figure 1 shows the CONSORT flow diagram for the study. Patients visiting participating practices were automatically enrolled (passive consent) and were informed of the SIMPle study through information leaflets and posters displayed in the waiting room.

Phase 1 started in June or July 2013 (depending on the practice), phase 2 started in September or October 2013, and phase 3 was introduced at the end of November 2013. The intervention ended Mar. 31, 2014.

The practice audit report met the Irish Medical Council requirement for general practitioners to maintain their professional competence. The Irish College of General Practitioners Research Ethics Committee reviewed and approved the intervention protocol.

\section{Outcome measure}

The primary outcome measure was proportion of prescriptions for recommended first-line antimicrobials for suspected urinary tract infection in intervention arms $\mathrm{A}$ and $\mathrm{B}$, relative to the control arm.

\section{Sample size}

The sample size was based on an absolute increase of $10 \%$ in the proportion of prescriptions for first-line antimicrobials in intervention arm A relative to control. A total of 920 patients with suspected urinary tract infection from 20 practices would give a power of $80 \%$ to detect a significant change in the proportion of patients receiving a first-line antimicrobial treatment in intervention arm A relative to the control arm (intraclass correlation $1 \%{ }^{5}$ ).

Table 1: Activities in the Supporting the Improvement and Management of Prescribing (SIMPle) study

\begin{tabular}{|c|c|c|c|}
\hline \multirow[b]{2}{*}{ Phase (after randomization) } & \multicolumn{3}{|c|}{ Study arm; activities* } \\
\hline & Intervention arm A & Intervention arm B & Control \\
\hline Phase 1: Baseline data collection & 1 & 1 & 1 \\
\hline Phase 2: Intervention period & 2,3 & $2,3,4$ & 1 \\
\hline Phase 3: Multimedia application & 5 & 5 & None \\
\hline Phase 4: Evaluation & 6 & 6 & 6 \\
\hline
\end{tabular}




\section{Data collection}

Throughout the study, data were extracted through the patient management software. We analyzed all antimicrobial prescriptions to compare overall prescribing and the prescribing of specific antimicrobials in each study arm. For each consultation with a physician, we extracted the patient's age, sex and medical card status. In Ireland, holders of medical cards receive free health care and medications. Entitlement to a medical card is based on income and age, and about one-third of the population under 70 years of age have a medical card. ${ }^{6}$ For patients with repeat consultations (within 30 days), only the first repeat consultation was considered. For each practice, the number of antimicrobial prescriptions per 100 practice consultations was calculated as a measure of practices with high and low prescribing. Other practice variables included the number of general practitioners, the presence of a practice nurse, the number of patients with a medical card, mean age of patients and mean age of general practitioners.

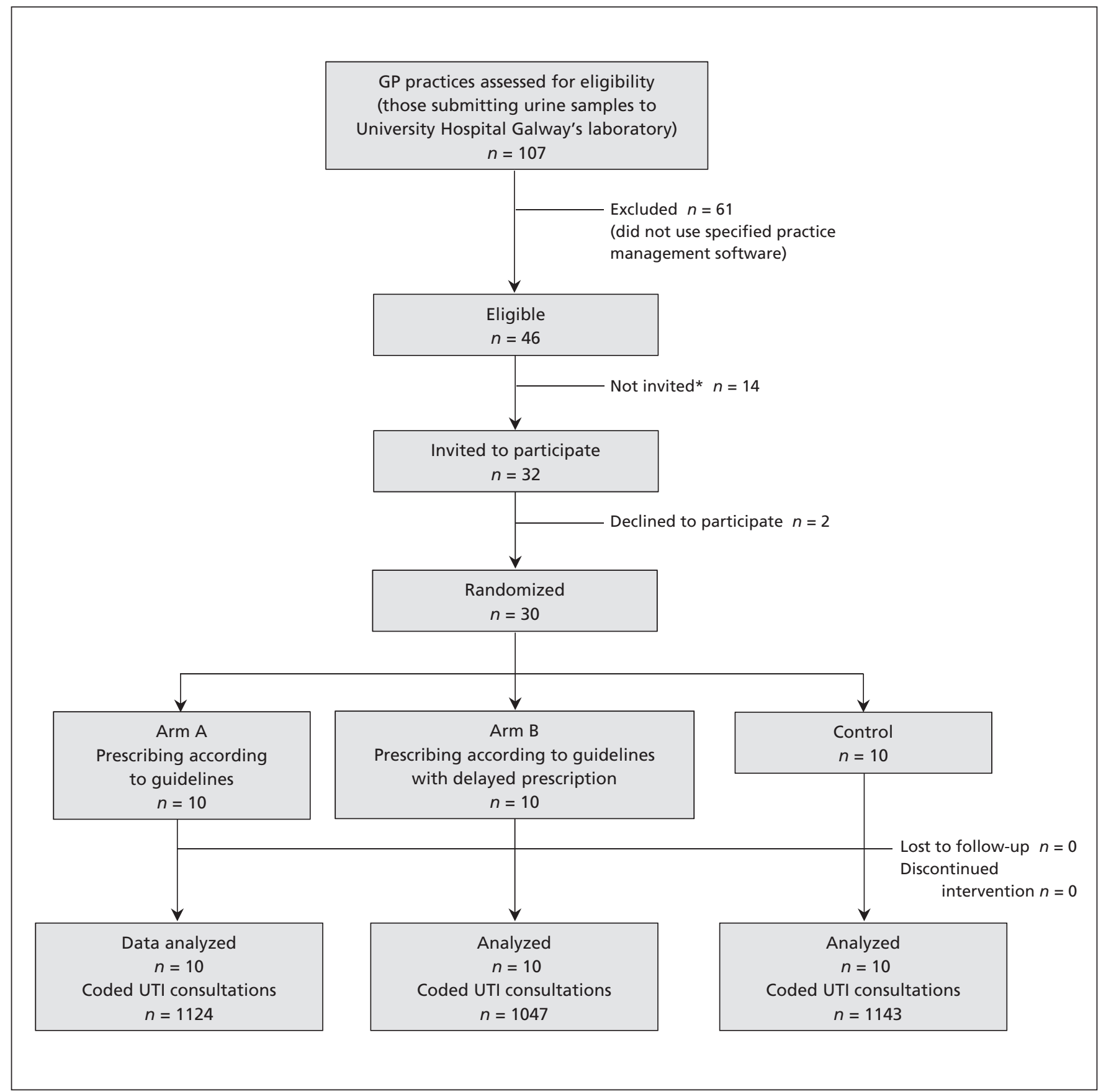

Figure 1: CONSORT flow diagram for the Supporting the Improvement and Management of Prescribing (SIMPle) study. GP = general practitioner. *Invitations were extended to practices that met the initial inclusion criterion until the target number of practices $(n=30)$ was reached. The remaining 14 practices met the eligibility criteria, but were not needed and hence were not invited. 


\section{Statistical analysis}

We took a population-averaged approach, using generalized estimating equation analysis with an exchangeable correlation structure, which allows a random intercept. ${ }^{7}$ This approach allowed clustering at the practice level and predicted first-line antimicrobial prescribing for urinary tract infection as a function of study arm, with adjustment for practice and patient characteristics. We ran similar models for each antimicrobial agent. We calculated adjusted odds ratios (ORs) for antimicrobial prescribing and associated $95 \%$ confidence intervals (CIs). We estimated intraclass correlation coefficients with the (xtgee) postestimation function (estat wcorrelation). Overall statistical analysis was performed with IBM SPSS version 21.0, and the generalized estimating equation analysis was performed with STATA 13 software.

\section{Deviations from protocol}

The primary objective of the intervention was to improve the prescribing of first-line antimicro- bials according to national guidelines. Trimethoprim and nitrofurantoin were both recommended as first-line treatment; however, the guidelines recommend using these first-line antimicrobials only below the resistance threshold of $20 \% .^{8}$ The prevalence of trimethoprim resistance was above this level, ${ }^{9}$ such that only nitrofurantoin remained as a recommended treatment. To be faithful to the protocol, both first-line treatment (i.e., trimethoprim or nitrofurantoin) and treatment with nitrofurantoin only were reported. In addition to the primary outcome, we determined changes in the frequency of prescribing (secondary objective), as well as specific other prescribing and reconsultation data to aid in the interpretation of the results. We based the power calculation on conservative estimates, according to active identification of patients by the general practitioners, similar to previous studies. ${ }^{5}$ Given the passive consent approach, we decided to adhere to the timelines set out in the protocol, which resulted in a larger sample size than planned.

Table 2: Overview of practices and patients for a study of antimicrobial prescribing for urinary tract infection

\begin{tabular}{|c|c|c|c|}
\hline \multirow[b]{2}{*}{ Characteristic } & \multicolumn{3}{|c|}{ Study arm; mean \pm SD* } \\
\hline & Intervention arm A & Intervention arm B & Control \\
\hline \multicolumn{4}{|l|}{ Practice } \\
\hline No. of GPs, median FTE (range) & $2.3(1.0-5.5)$ & $2.0(1.0-5.0)$ & $2.0(1.0-4.5)$ \\
\hline Time in practice, $y r$ & $18.7 \pm 12.5$ & $14.8 \pm 11.4$ & $16.9 \pm 8.8$ \\
\hline No. of practice contacts per yr & $14810 \pm 10169$ & $15464 \pm 12950$ & $12820 \pm 7661$ \\
\hline $\begin{array}{l}\text { No. of antimicrobial prescriptions } \\
\text { per } 100 \text { practice contacts }\end{array}$ & $14.1 \pm 4.6$ & $11.0 \pm 4.8$ & $12.2 \pm 4.1$ \\
\hline \multicolumn{4}{|l|}{ Study } \\
\hline \multicolumn{4}{|l|}{ No. of UTI consultations } \\
\hline Baseline (before interventiont) & 381 & 309 & 360 \\
\hline During interventiont & 743 & 738 & 783 \\
\hline Subtotal, baseline + interventiont & 1124 & 1047 & 1143 \\
\hline Follow-up & 211 & 241 & 441 \\
\hline Repeat consultations (interventiont) & 18 & 14 & 36 \\
\hline \multicolumn{4}{|l|}{ During intervention period } \\
\hline $\begin{array}{l}\text { No. (\%) of consultations with urine } \\
\text { samples }\end{array}$ & $350(47.1)$ & $380(51.5)$ & $377(48.1)$ \\
\hline No. $(\%)$ of samples with growth & $231(66.0)$ & $248(65.3)$ & $239(63.4)$ \\
\hline \multicolumn{4}{|l|}{ Consultations (by practice) } \\
\hline Age of patients, yr & $56.3 \pm 3.3$ & $51.5 \pm 11.4$ & $54.1 \pm 7.7$ \\
\hline Sex of patients, $\%$ male & $12.0 \pm 6.6$ & $12.4 \pm 7.8$ & $8.3 \pm 5.6$ \\
\hline$\%$ of patients with medical card & $68.1 \pm 14.4$ & $62.1 \pm 17.5$ & $55.3 \pm 18.8$ \\
\hline
\end{tabular}




\section{Results}

\section{Demographic characteristics}

A total of 30 practices, accounting for 71 general practitioners, were randomly assigned to the 3 study arms (Table 1 and Figure 1). Over the 9-month study period, we recorded a total of 3314 consultations for urinary tract infection (Table 2), evenly spread among the 3 arms over the entire intervention period. Nearly $32 \%(n=$ $1050)$ of the consultations were recorded in the baseline period (3 mo) and $68.3 \%$ (2264) during the intervention period $(6 \mathrm{mo})$. The proportion of consultations in which an antimicrobial agent was prescribed was $67.0 \%(704 / 1050)$ in the baseline period and $73.5 \%(1664 / 2264)$ in the intervention period.

The consultations for urinary tract infection involved mostly women (89.4\% [2963/3314]) and patients with a medical card (66.0\% [2187/3314]). The mean age of patients was 56.1 (standard deviation 20.7) years. The total number of patients included in the analysis was 2560 , with a mean of 110 (standard deviation 13.6) patients per practice (range 34 to 328). Table 2 presents an overview of practice and consultation characteristics.

\section{Prescribing practices}

Antimicrobial prescribing did not differ among groups during the baseline period, and a first-line antimicrobial agent was prescribed in $45.4 \%$ to $49.8 \%$ of the consultations for urinary tract infection (Table 3). At the end of the intervention period, the rate of first-line prescribing was $68.2 \%$ in arm A (absolute increase 22.8\%),
$66.5 \%$ in arm B (absolute increase 16.7\%) and $44.1 \%$ in the control group (absolute decrease $1.7 \%)$. Relative to the control group, the absolute increase in first-line prescribing was $24.5 \%$ in arm $\mathrm{A}$ and $18.4 \%$ in arm B.

When only nitrofurantoin was considered, the absolute increase in prescribing was $37.5 \%$ in arm $\mathrm{A}$ and $32.7 \%$ in arm B, whereas prescribing remained stable in the control arm (absolute difference $0.9 \%$ ). However, the absolute change in percentage of consultations with prescribing of any antimicrobial was a $15.3 \%$ increase in arm A, a $5.9 \%$ increase in arm B and a $2.1 \%$ decline in the control arm. With consideration of the total percentage of antimicrobial prescribing in the intervention arms, the increase in nitrofurantoin prescribing came through replacement of trimethoprim (about 15\%) and co-amoxyclav (amoxicillin and clavulanic acid; less than 10\%) but not quinolones (see Appendix 1, available at www.cmaj.ca/lookup /suppl/doi:10.1503/cmaj.150601/-/DC1).

The effect of the intervention was calculated as an OR in a logistic generalized estimating equation model (Table 4). The adjusted OR was 2.7 (95\% CI 1.8 to 4.1) for intervention arm A and 2.0 (95\% CI 1.3 to 3.0) for intervention arm B. The adjusted overall OR of 2.3 (95\% CI 1.7 to 3.2) means that a patient visiting an intervention practice with symptoms suggestive of urinary tract infection was 2.3 times more likely to receive a prescription for a first-line antimicrobial than a similar patient visiting a control practice.

The adjusted odds that a patient would receive a prescription for nitrofurantoin were 4.5 (95\% CI 2.7 to 7.3$)$ in arm A and 3.5 (95\% CI

\begin{tabular}{|c|c|c|c|c|c|c|c|c|}
\hline \multirow[b]{2}{*}{ Variable } & \multicolumn{8}{|c|}{ Study arm; timing; measure $(95 \% \mathrm{Cl})$} \\
\hline & & Baseline & Intervention period & Baseline & Intervention period & & Baseline & Intervention period \\
\hline No. of consultations & & 381 & 743 & 309 & 738 & & 360 & 783 \\
\hline$\%$ of prescriptions & 45.4 & (40.4 to 50.4$)$ & 68.2 (64.9 to 71.6$)$ & 49.8 (44.2 to 55.4$)$ & 66.5 (63.1 to 69.9$)$ & 45.8 & (40.7 to 51.0 ) & 44.1 (40.6 to 47.6 ) \\
\hline Absolute difference* & & & 22.8 (16.6 to 29.0$)$ & & 16.7 (9.9 to 23.5$)$ & & & $-1.7 \quad(-4.7$ to 8.1$)$ \\
\hline Difference (v. control) & & & 24.5 (21.9 to 27.1$)$ & & 18.4 (16.0 to 20.8$)$ & & & NA \\
\hline \multicolumn{9}{|l|}{ Nitrofurantoin } \\
\hline$\%$ of prescriptions & 26.8 & (22.3 to 31.2$)$ & 64.3 (60.9 to 67.8 ) & 31.1 (25.9 to 36.3 ) & 63.8 (60.4 to 67.3$)$ & 35.0 & (30.1 to 40.0 ) & 35.9 (32.5 to 39.3$)$ \\
\hline Absolute difference* & & & 15.3 (9.4 to 21.2$)$ & & $5.9(-0.3$ to 12.1$)$ & & & $-2.1 \quad(-3.9$ to 8.1$)$ \\
\hline
\end{tabular}


1.9 to 6.3 ) in arm B. To identify the effect of increased prescribing, a model was run estimating the odds of receiving an antimicrobial depending on the arm. This model showed that patients in arm A had a higher chance of receiving an antimicrobial than patients in the control group (adjusted OR 2.2, 95\% CI 1.2 to 4.0), but this was not so for patients in arm B (adjusted OR 1.4, $95 \%$ CI 0.9 to 2.1). No practice factors or other intervention approaches (e.g., multimedia applications) had a significant effect on the outcome.

\section{Overall antimicrobial prescribing and sustainability}

Figure 2 shows, for practice contacts with prescribing of any antimicrobial, the percentage in which nitrofurantoin was prescribed. For arm A, there was an increase from $26.8 \%$ at baseline to $64.3 \%$ during the intervention, which was sustained at $63.5 \%$ during follow-up. Similarly, for arm B there was an increase from $31.1 \%$ at baseline to $63.8 \%$ during the intervention, with only a slight drop, to $57.3 \%$, during follow-up. Prescribing of nitrofurantoin in the control arm was about $35 \%$ both before and during the intervention, increasing to $47.8 \%$ during the follow-up period.

\section{Coding of consultations}

Given that nitrofurantoin is prescribed almost exclusively for patients with suspected urinary tract infection, the data for nitrofurantoin provided a basis for estimating completeness of implementation of coding. For each study arm and each practice, we examined the percentage of nitrofurantoin prescriptions accounted for by UTI-coded consultations (Table 5). Between $40 \%$ and $50 \%$ of the nitrofurantoin prescriptions were coded during the intervention, and this proportion dropped to less than $30 \%$ during follow-up.

\section{Repeat consultations}

A total of 68 repeat consultations were identified during the intervention period, and generalized estimating equation analysis showed no difference in repeat consultation between the intervention and control arms (arm A adjusted OR 0.5, 95\% CI 0.2 to 1.3 ; arm B adjusted OR 0.4, 95\% CI 0.1 to 1.1). However, patients who received nitrofurantoin during the index visit were less likely to have a repeat consultation (adjusted OR $0.6,95 \%$ CI 0.3 to 0.99 ). No differences in repeat consultation were observed when other antimicrobials were prescribed at the index visit.

\section{Interpretation}

The primary aim of the SIMPle study was to improve the quality of antimicrobial prescribing according to guidelines. An absolute increase of $20 \%$ was achieved for practices in the intervention arms, and patients attending an intervention practice were twice as likely to receive a prescription for a first-line antimicrobial for their urinary tract infection as those attending a control practice.

The focus of our intervention was on increasing the proportion of antimicrobial prescriptions for nitrofurantoin. In line with recent updates to guidelines on the treatment of urinary tract infections,,$^{10}$ the use of quinolones is discouraged, to retain quinolones as a viable alternative if firstline treatment fails. With trimethoprim resistance higher than $20 \%$, prescribing of nitrofurantoin was an alternative outcome. Patients visiting an intervention practice were up to 5 times more likely to receive a prescription for nitrofurantoin than any other antimicrobial. However, the improved quality of prescribing must be put into the context of its unintended effect, an increase in actual antimicrobial prescriptions. Research has shown that the nature of complex systems,

Table 4: Adjusted* effect of interventions by outcome

\begin{tabular}{|lccccc|}
\hline & \multicolumn{5}{c}{ Antimicrobial; OR $(95 \% \mathrm{Cl})$} \\
\cline { 2 - 6 } Variable & First-line & Nitrofurantoin & Quinolone & Trimethoprim & Co-amoxyclav \\
\hline Control & Reference & Reference & Reference & Reference & Reference \\
\hline Arm A & $2.7(1.8$ to 4.1$)$ & $4.5(2.7$ to 7.3$)$ & $0.6(0.3$ to 1.00$)$ & $0.3(0.1$ to 0.6$)$ & $0.4(0.3$ to 0.7$)$ \\
\hline Arm B & $2.0(1.3$ to 3.0$)$ & $3.5(1.9$ to 6.3$)$ & $0.7(0.3$ to 1.4$)$ & $0.2(0.1$ to 0.3$)$ & $0.3(0.2$ to 0.6$)$ \\
\hline Age, per yr & $1.0(0.99$ to 1.0$)$ & $1.0(1.0$ to 1.0$)$ & $1.0(1.0$ to 1.0$)$ & $1.0(0.98$ to 1.0$)$ & $0.99(0.99$ to 1.0$)$ \\
\hline Sex, male & $0.5(0.4$ to 0.7$)$ & $0.6(0.4$ to 0.8$)$ & $2.4(1.5$ to 3.9$)$ & $0.7(0.4$ to 1.2$)$ & $0.9(0.5$ to 1.7$)$ \\
\hline Medical card & $1.1(0.9$ to 1.3$)$ & $1.0(0.8$ to 1.3$)$ & $0.7(0.4$ to 0.98$)$ & $1.2(0.9$ to 1.5$)$ & $1.1(0.8$ to 1.5$)$ \\
\hline ICC & 0.048 & 0.045 & 0.035 & 0.031 & 0.005 \\
\hline $\begin{array}{l}\text { Note: } \mathrm{Cl}=\text { confidence interval, ICC }=\text { intraclass correlation (measure of resemblance among practices), OR }=\text { odds ratio. } \\
\text { * ORs were adjusted for age, sex, medical card status and number of antimicrobial prescriptions per } 100 \text { practice consultations. }\end{array}$ \\
\hline
\end{tabular}


such as general practices, where many interrelated factors influence antimicrobial prescribing, makes it difficult to predict the results of interventions. Although our intervention did not show results opposite to those we anticipated, the actual intervention effect was inflated. We can only speculate that a more stepwise approach, in which successful implementation of one behavioural change (improved prescribing) would be followed by a next step in behavioural change (limitation on prescribing), might be more appropriate.

From a societal perspective, the potential negative effect of increased use of antimicrobials may be mitigated if the increase involves nitrofurantoin. After extensive worldwide use of this drug for more than 50 years, there has been little evidence of acquired resistance to nitrofurantoin, and the use of this agent does not appear to predispose patients to resistance. ${ }^{11,12}$ Therefore, nitrofurantoin may be less harmful than and much preferred over other antimicrobials. ${ }^{13,14}$ Also, the lower number of repeat consultations among patients for whom nitrofurantoin was pre-

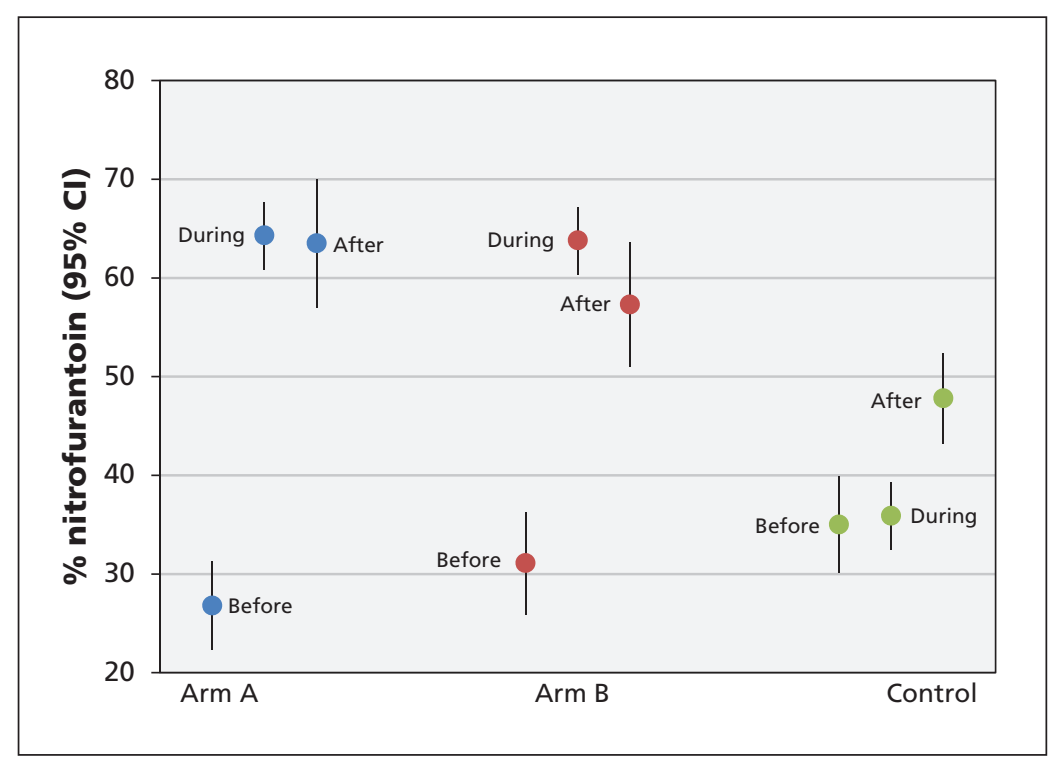

Figure 2: Percentage of practice contacts in which nitrofurantoin was prescribed (for practice contacts with prescribing of any antimicrobial), before, during and after the intervention in each study arm. $\mathrm{Cl}=$ confidence interval.

Table 5: Proportion of UTI-coded nitrofurantoin prescriptions

\begin{tabular}{|lccc|}
\hline & \multicolumn{3}{c|}{ Study arm; \% UTI-coded } \\
\cline { 2 - 4 } Time frame & Arm A & Arm B & Control \\
\hline Baseline & 47.4 & 40.5 & 50.0 \\
\hline During intervention & 41.0 & 46.5 & 44.9 \\
\hline Follow-up & 18.0 & 21.8 & 30.8 \\
\hline Note: UTI = urinary tract infection. & & & \\
\hline
\end{tabular}

scribed may support the finding that nitrofurantoin does not predispose patients to resistance.

Analysis of the 5-month follow-up data showed that the behavioural change initiated by the intervention was sustained and became embedded in clinical practice. However, coding of urinary tract infection consultations dropped after the intervention and thus was not embedded as a behavioural change. The potential of coding to provide practice-specific information, and thereby to facilitate audit reports, may need further emphasis.

In a cluster randomized controlled trial (RCT) in Norwegian general practice, which aimed to change the use of antimicrobials for urinary tract infection, patients received educational material and general practitioners received computerbased decision support and reminders. ${ }^{15}$ The RCT was delivered passively, without support related to guidelines. Despite the advanced design of this study's intervention, the effect was limited. Another Norwegian study to limit antimicrobial prescribing for respiratory infections used an RCT design to implement multifaceted academic detailing as part of continuing medical education. ${ }^{16}$ In this RCT, there was better adherence to guidelines and a reduction in antimicrobial prescribing, which suggests that changing the prescribing behaviour of general practitioners should be integrated into their continuing education. A recent simple and low-cost RCT showed great promise in reducing inappropriate prescribing through the use of public commitment letters from general practitioners, without additional support; there was an absolute reduction of nearly $20 \%$ in inappropriate prescribing of antimicrobials, which did not diminish over the 1-year duration of the study. ${ }^{17}$ Buy-in from general practitioners through public commitment or appropriate incentives and integration of behaviour change as part of continuing education should be considered for future interventions. ${ }^{18}$

\section{Limitations and strengths}

This study had some limitations. First, the increase in overall prescribing of antimicrobials for urinary tract infection was unexpected, and it was not possible to conclude whether this was clinically appropriate or an unwanted consequence. This finding merits further study. Second, delayed prescribing could be identified only indirectly. For a future study, the option of recording a prescription as delayed in the patient management software should be considered; this option might even serve as a reminder. Other limitations included the study's limited geographic range (leading to limited external validity), the relatively small number of practices and 
the input of a research team for the duration of the study.

This study also added value to existing evidence in several respects. An audit report, the key element in changing prescribing behaviour, included practice-specific information. Passive enrolment through coded consultations resulted in a larger sample size than anticipated, which reflects the simplicity of this study for general practitioners and serves as an example of integrating research into practice. The sustainability of the intervention was reflected by continued first-line prescribing at 5 months after the intervention. The success of the SIMPle study has garnered the interest of the Irish College of General Practice, and a national rollout is planned.

\section{Conclusion}

Clear, contextualized, practice-specific information in the form of feedback reports is a highly efficient method to investigate and change the antimicrobial prescribing behaviour of general practitioners. Research and practice can be integrated through novel data collection methods that do not require active recruitment. The SIMPle study, which involved a complex intervention including audit and feedback reports combined with reminders, improved the quality of antimicrobial prescribing for urinary tract infection in Irish general practice.

\section{References}

1. Gulland A. Antimicrobial resistance is now widespread, warns WHO. BMJ 2014;348:g3062.

2. Antimicrobial resistance: global report on surveillance. Geneva: World Health Organization; 2014.

3. Duane S, Callan A, Galvin S, et al. Supporting the improvement and management of prescribing for urinary tract infections (SIMPle): protocol for a cluster randomized trial. Trials 2013;14:441.

4. Guidelines for antimicrobial prescribing in primary care in Ireland. Dublin: Health Service Executive and Royal College of Physicians of Ireland; 2012. Available: www.antibioticprescribing.ie/ (accessed 2015 July).

5. Vellinga A, Tansey S, Hanahoe B, et al. Trimethoprim and ciprofloxacin resistance and prescribing in urinary tract infection associated with Escherichia coli: a multilevel model. J Antimicrob Chemother 2012;67:2523-30.

6. Consumers survey: the private health insurance market in Ireland 2014. Dublin: Health Insurance Authority; 2014. Available: www.hia.ie/sites/default/files/HIA_Consumer\%20Survey 2014 Final.pdf (accessed 2015 Sept. 29)

7. Twisk JWR. Applied multilevel analysis: a practical guide Cambridge (UK): Cambridge University Press; 2006.

8. Gupta K, Hooton TM, Naber KG, et al. International clinical practice guidelines for the treatment of acute uncomplicated cystitis and pyelonephritis in women: a 2010 update by the Infectious Diseases Society of America and the European Society for Microbiology and Infectious Diseases. Clin Infect Dis 2011;52:e103-20.

9. Vellinga A, Cormican M, Hanahoe B, et al. Antimicrobial management and appropriateness of treatment of urinary tract infection in general practice in Ireland. BMC Fam Pract 2011;12:108.

10. Grigoryan L, Trautner BW, Gupta K. Diagnosis and management of urinary tract infections in the outpatient setting: a review. JAMA 2014;312:1677-84.
11. Brumfitt W, Hamilton-Miller JM. Efficacy and safety profile of long-term nitrofurantoin in urinary infections: 18 years' experience. J Antimicrob Chemother 1998;42:363-71.

12. Vellinga A, Cormican M, Hanahoe B, et al. Predictive value of antimicrobial susceptibility from previous urinary tract infection in the treatment of re-infection. Br J Gen Pract 2010;60: 511-3.

13. Cunha BA, Schoch PE, Hage JR. Nitrofurantoin: preferred empiric therapy for community-acquired lower urinary tract infections. Mayo Clin Proc 2011;86:1243-4, author reply 1244

14. McKinnell JA, Stollenwerk NS, Jung CW, et al. Nitrofurantoin compares favorably to recommended agents as empirical treatment of uncomplicated urinary tract infections in a decision and cost analysis. Mayo Clin Proc 2011:86:480-8

15. Flottorp S, Oxman AD, Havelsrud K, et al. Cluster randomised controlled trial of tailored interventions to improve the management of urinary tract infections in women and sore throat. BMJ 2002;325:367.

16. Gjelstad S, Hoye S, Straand J, et al. Improving antibiotic prescribing in acute respiratory tract infections: cluster randomised trial from Norwegian general practice (prescription peer academic detailing (Rx-PAD) study). BMJ 2013;347: f4403.

17. Meeker D, Knight TK, Friedberg MW, et al. Nudging guideline-concordant antibiotic prescribing: a randomized clinical trial. JAMA Intern Med 2014;174:425-31.

18. Arnold SR, Straus SE. Interventions to improve antibiotic prescribing practices in ambulatory care. Cochrane Database Syst Rev 2005;(4)CD003539.

Affiliations: Discipline of General Practice (Vellinga, Galvin, Duane, Callan, Murphy) and Discipline of Bacteriology (Vellinga, Cormican), School of Medicine, and JE Cairnes School of Business and Economics (Domegan), National University of Ireland, Galway, Ireland; Department of Pharmacology and Therapeutics (Bennett), Trinity College Dublin, Dublin, Ireland; Department of Medical Microbiology (Cormican), University Hospital Galway, Galway, Ireland

Contributors: Akke Vellinga conceived the study; led protocol development, study implementation, data management, statistical analysis and interpretation of the data; and drafted the manuscript. Sandra Galvin, Sinead Duane and Aoife Callan contributed to protocol and intervention development, design of the intervention materials, and data collection and management. Sandra Galvin and Sinead Duane delivered the intervention to all practices and liaised with practices on data collection. Kathleen Bennett contributed to protocol development, study implementation and data interpretation. Martin Cormican provided microbiologic support and contributed to protocol development. Christine Domegan contributed to protocol development, intervention structure and content design. Andrew Murphy conceived the study and acted as principal investigator. All authors approved the final version to be published and agreed to act as guarantors of the work.

Funding: This study was funded by the Health Research Board of Ireland under Interdisciplinary Capacity Enhancement Award ICE2011-10. Additional funding was obtained for the multimedia applications through a 2012 grant from the Knowledge Exchange and Dissemination Scheme from the Health Research Board.

Data sharing: All anonymized data gathered during this study are available to other researchers upon request (by contacting the first author). Additional approval from ethical and/or research committees may be needed for subsequent use of these data.

Acknowledgements: The authors would like to acknowledge the participation and support of the staff of the 30 general practices that participated in the study and all patients who participated in the provision of data. The authors also acknowledge the staff of the University Hospital Galway laboratory. Practice data collection was carried out through the Irish Primary Care Research Network. 